\title{
Dome C site testing: long term statistics of integrated optical turbulence parameters at ground level
}

\author{
E. Aristidi, A. Agabi, E. Fossat, A. Ziad, L. Abe, E. Bondoux, \\ G. Bouchez, Z. Challita, F. Jeanneaux, D. Mékarnia, D. Petermann \\ and C. Pouzenc
}

Laboratoire Lagrange, Université de Nice, Parc Valrose, F-06108 Nice Cedex 2

\begin{abstract}
We present long term site testing statistics based on DIMM and GSM data obtained at Dome C, Antarctica. These data have been collected on the bright star Canopus since the end of 2003. We give values of the integrated turbulence parameters in the visible (wavelength $500 \mathrm{~nm}$ ). The median value we obtained for the seeing are $1.2 \operatorname{arcsec}, 2.0 \operatorname{arcsec}$ and $0.8 \operatorname{arcsec}$ at respective elevations of $8 \mathrm{~m}, 3 \mathrm{~m}$ and $20 \mathrm{~m}$ above the ground. The isoplanatic angle median value is 4.0 arcsec and the median outer scale is $7.5 \mathrm{~m}$. We found that both the seeing and the isoplanatic angle exhibit a strong dependence with the season (the seeing is larger in winter while the isoplanatic angle is smaller).
\end{abstract}

Keywords. site testing, atmospheric effects, optical turbulence

\section{Introduction}

The AstroConcordia program has been, up to now, dedicated to the qualification of the site of Dome C, Antarctica for astronomical purposes. After almost 10 years of operation since the first results (Aristidi et al. 2003) we could measure long terms characteristics of the turbulent atmosphere. In this contribution we present the results of a decade of turbulence monitoring. We give statistics of the seeing, the isoplanatic angle, and the outer scale during the polar winter and summer. This paper is indeed an update of previously published work: Aristidi et al. (2009) and Ziad et al. (2008).

\section{Results}

All results presented here come from telescope-based instruments which observe a bright star in the visible and perform continuous measurements of the seeing, the isoplanatic angle and the outer scale. These instruments named DIMM, Thetameter and GSM are described in previous papers (Aristidi et al. 2005, 2009, Ziad et al. 2008). Several DIMM were placed at different elevations to sample the seeing inside the turbulent surface layer. One was at ground level (elevation $\mathrm{h} \simeq 3 \mathrm{~m}$ ) as a part of GSM, one at $\mathrm{h} \simeq 8 \mathrm{~m}$ (this DIMM is taken as the standard one), and a DIMM was placed over the top of the calm building of Concordia at $\mathrm{h} \simeq 20 \mathrm{~m}$ in 2005 and 2012 .

Statistics for the three parameters are given in Table 1. A big difference is observed between the summer and the winter for the seeing and the isoplanatic angle. The latter is sensitive to high altitude turbulent layers and decrease in winter as the polar vortex drives strong winds in the upper atmosphere.

The seeing is dominated by a strongly turbulent surface layer (SL) whose height is around $30 \mathrm{~m}$. This layer is partly caused by a huge thermal gradient near the ground in 


\begin{tabular}{l|c|c|c} 
& Winter (Apr - Sept) & Summer (Dec - Jan) & Total \\
\hline Seeing $h=3 \mathrm{~m}[\operatorname{arcsec}]$ & $2.4[1.8-3.2]$ & $1.0[0.7-1.3]$ & $2.0[1.4-2.8]$ \\
Seeing $h=8 \mathrm{~m}[\operatorname{arcsec}]$ & $1.7[1.0-2.4]$ & $0.7[0.4-0.8]$ & $1.2[0.6-1.9]$ \\
Seeing $h=20 \mathrm{~m}[\operatorname{arcsec}]$ & $0.8[0.4-1.5]$ & - & $0.8[0.4-1.5]$ \\
Isop. angle [arcsec] & $3.2[2.3-4.4]$ & $6.4[4.5-8.5]$ & $4.0[2.7-5.8]$ \\
Outer scale [m] & - & - & $7.5[5.1-11.3]$ \\
\hline
\end{tabular}

Table 1. Statistics of the seeing at 3 elevations, the isoplanatic angle and the outer scale (Von-Kàrman model). Median value is given as well as the 1st and 3rd quartiles (between []).
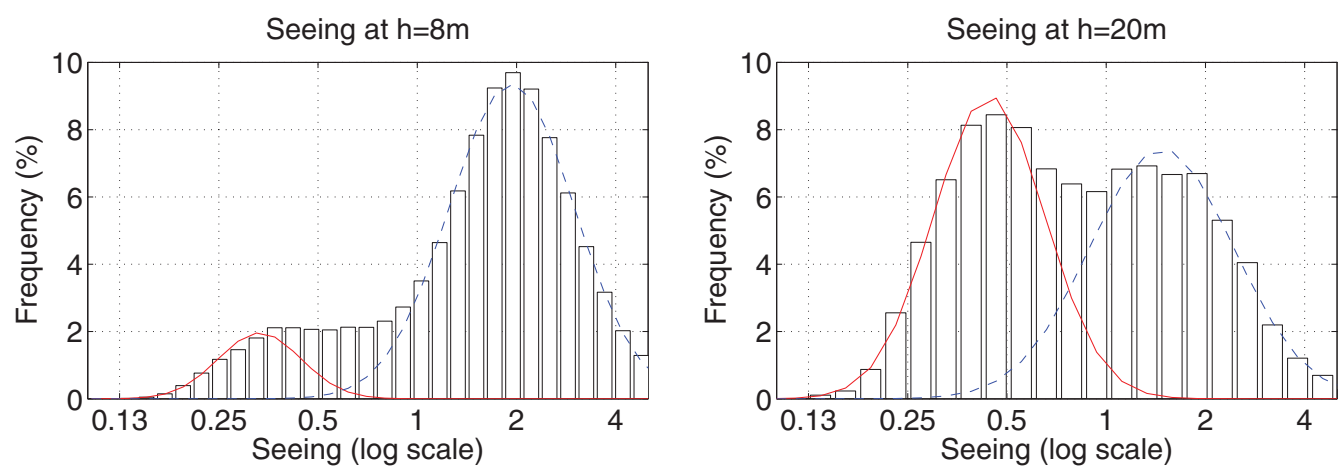

Figure 1. Left: Seeing histogram at an elevation of $h=8 m$ for the period April to September. Right: Idem for $h=20 \mathrm{~m}$ from available data in 2005 and 2012. Red (solid) and blue (dashed) lines are Gaussian fit of the two bumps. The area under them gives the fraction of time spent by the DIMM inside (dashed line) or outside (solid line) the SL. At $h=20 m$ the DIMM is in the free atmosphere $50 \%$ of the time $(12 \%$ at $h=8 \mathrm{~m})$.

winter, and disappears partly in summer (Aristidi et al. 2009). The winter seeing is then highly dependent of the height of the telescope pupil. Fig. 1 presents the winter seeing histograms at $h=8 \mathrm{~m}$ and $20 \mathrm{~m}$. The two-bumps structure corresponds to situations where the DIMM is either inside or outside the SL, and the area under each bump gives the fraction of time spent in each situation. At $h=20 \mathrm{~m}$, the telescope is half of the time in the free atmosphere (FA) and this percentage will increase with $h$.

Regarding the outer scale, we here confirm the preliminary value of $7.5 \mathrm{~m}$ published in Ziad et al. 2008). The measurements were made at ground level at $h=3 \mathrm{~m}$. No particular variability was observed between the summer and the winter.

\section{Conclusion}

In this paper we gave an update of the statistics of optical turbulence parameters and confirm the general trends published before: poor conditions at ground level in winter, but superb situation at an elevation of a few tens of meters above the snow where the FA can be attained during a significant amount of time. In the FA the median seeing is around $0.4^{\prime \prime}$ (Aristidi et al. 2009).

\section{References}

Aristidi, E., Agabi, A., Vernin, J., et al. 2003, A\& A, 406, L19

Aristidi, E., Agabi, A., Fossat, E., et al. 2005, A\& $A$, 444, 651

Aristidi, E., Fossat, E., Agabi, A., et al. 2009, A\& A, 499, 955

Ziad, A., Aristidi, E., Agabi, et al. 2008, A\& A, 491, 917 